\title{
Simulation of transition radiation based beam imaging from tilted targets
}

\author{
L. G. Sukhikh, ${ }^{1, *}$ G. Kube, ${ }^{2}$ and A.P. Potylitsyn ${ }^{1}$ \\ ${ }^{1}$ Tomsk Polytechnic University, Tomsk 634050, Russia \\ ${ }^{2}$ Deutsches Elektronen-Synchrotron (DESY), Hamburg 22607, Germany \\ (Received 13 May 2016; revised manuscript received 20 January 2017; published 15 March 2017) \\ Transverse beam profile diagnostics in linear electron accelerators is usually based on direct imaging of a \\ beam spot via visible transition radiation. In this case the fundamental resolution limit is determined by \\ radiation diffraction in the optical system. A method to measure beam sizes beyond the diffraction limit is \\ to perform imaging dominated by a single-particle function (SPF), i.e. when the recorded image is \\ dominated not by the transverse beam profile but by the image function of a point source (single electron). \\ Knowledge of the SPF for an experimental setup allows one to extract the transverse beam size from an SPF \\ dominated image. This paper presents an approach that allows one to calculate two-dimensional SPF \\ dominated beam images, taking into account the target inclination angle and the depth-of-field effect. In \\ conclusion, a simple fit function for beam size determination in the case under consideration is proposed \\ and its applicability is tested under various conditions.
}

DOI: $10.1103 /$ PhysRevAccelBeams.20.032802

\section{INTRODUCTION}

Next generation electron accelerators, for example the linear collider projects ILC or CLIC, linear accelerator (linac) based free electron lasers (FELs), and electron beam driven plasma accelerators, require electron beam sizes in the order of $10 \mu \mathrm{m}$ down to submicrometers. In addition to the challenge of generating these small beams, it must be possible to monitor such small beam sizes with sufficiently high resolution to prove the achievement of the required beam parameters.

Transverse beam profile diagnostics based on transition radiation is a standard technique at electron linacs. This radiation is emitted when a beam of charged particles crosses the boundary between two media with different optical properties. The radiation is generated in a nearly instantaneous process directly at the screen boundary, and it has a lobe-shape pattern with a characteristic opening angle as defined by the beam energy. Neglecting coherent effects, transition radiation has a linear response and a rather high light output that makes its application suitable for sub-nC particle beam diagnostics in modern accelerators. Due to practical reasons, the visible spectral part of this radiation (so-called optical transition radiation or OTR), emitted in the direction of the specular reflection is used for transverse beam profile imaging [1]. In an intuitive picture, backward OTR generation can simply be considered as a reflection of the particle electromagnetic field by the target surface, i.e. the

\footnotetext{
*Sukhikh@tpu.ru

Published by the American Physical Society under the terms of the Creative Commons Attribution 4.0 International license. Further distribution of this work must maintain attribution to the author(s) and the published article's title, journal citation, and DOI.
}

source is represented as pseudophotons that are converted to real photons due to the momentum transfer from the target. The working principle of an OTR beam profile monitor is such that the beam spot is imaged via OTR onto a spatial resolving detector using a conventional optical system.

While OTR based diagnostics is widely used in electron linacs (see e.g. Refs. [2-5]) covering an energy range from $10 \mathrm{keV} \mathrm{[6]} \mathrm{up} \mathrm{to} 30 \mathrm{GeV}$ [7], it has also been applied in proton accelerators [8,9] and for heavy ion beam diagnostics [10].

In the frame of classical optics, the fundamental resolution in the imaging process is usually discussed in terms of the point-spread function (PSF) which is solely defined by the optical system in use: in the case of image generation with an ideal thin lens for example, the PSF is expressed by the wellknown Airy pattern. For imaging with OTR however, the image of a single electron caused by an ideal thin lens does not depend only on the optical system properties but also on the characteristics of OTR radiation which are affected by particle beam and target properties as expressed by the Lorentz factor $\gamma$ and the dielectric constant $\varepsilon$. Because of the similarity to the classical PSF and in order to avoid misunderstanding, in the following the OTR single particle image will be denoted the single-particle function (SPF).

First calculations of the OTR SPF were carried out by Lebedev in Ref. [11] who showed that the charged particle SPF is a double-lobe function that influences the OTR monitor spatial resolution. In Ref. [12] Castellano and Verzilov investigated the SPF characteristics in the case of ideal imaging. Further investigations were carried out by Potylitsyn [13] who studied the influence of the prewave zone effect on the OTR SPF, and by Xiang and Huang [14] who investigated some aspects of the beam imaging resolution. The first systematic resolution study of an OTR based beam profile monitor in view of a dedicated application was made by Kube in Ref. [15] who 
investigated additionally the influence of optical system imperfections on the SPF. Imaging with coherent and incoherent transition radiation at flat and rough target surfaces was discussed by Stupakov [16]. Following these references the SPF has a double-lobe structure which is defined by the radiation wavelength $\lambda$, the acceptance angle or numerical aperture of the optical system $\theta_{m}$, and the alignment accuracy. For the condition of ideal imaging the OTR monitor resolution can be expressed as [15]

$$
R=1.12 \frac{\lambda M}{\theta_{m}}
$$

Here $M$ denotes the magnification factor of the optical system. The resolution Eq. (1) is in close analogy to that defined in classical optics by the first minimum of the Airy pattern which is about a factor of 2 smaller [15]. For a typical optical system $\left(\theta_{m}=0.1\right)$ and visible light $(\lambda=500 \mathrm{~nm})$ the OTR monitor resolution is given by $R / M=5.6 \mu \mathrm{m}$, thus making it difficult to measure smaller beam sizes.

In order to enable measurements of micron or even submicron beam sizes it is possible to improve the resolution by decreasing the wavelength of observation, see Eq. (1). In Refs. [17,18] it was demonstrated that backward transition radiation in the extreme ultraviolet (EUV) spectral region $(\lambda \approx 19 \mathrm{~nm})$ can be used for beam profile imaging. While EUV transition radiation has sufficient intensity for beam diagnostics applications, the main disadvantage in this spectral region is the strong radiation absorption in any material, making it necessary to install the entire monitor setup in a vacuum system. Maintaining the advantages of beam diagnostics with visible light, it is possible to achieve a resolution beyond the diffraction limit imposed by Eq. (1) even in the visible spectral region. In the case of SPF dominated imaging, the recorded image is dominated by the resolution function of a point source, and with exact knowledge of the SPF it is possible to extract beam size information. In a recent experiment by Karataev et al., OTR SPF dominated beam images were measured at ATF-II facility at KEK (Tsukuba, Japan) [19]. However, the observed double-lobe structures were significantly wider than those predicted theoretically. As a result, beam images were seriously distorted, i.e. despite the large lens numerical aperture $\left(\theta_{m}=0.24\right)$ nonGaussian beam shapes were imaged even for beams with vertical root-mean-square (rms) sizes as large as $4 \mu \mathrm{m}$.

In Ref. [20] a fit function and calibration procedure was proposed that allows one to obtain beam sizes in the case of SPF dominated OTR beam images. In a subsequent publication [21] the authors presented a modified fit function and additional measurements of SPF dominated beam images from a flat beam (i.e. a beam with small vertical and large horizontal size). It was shown that such a beam is imaged as a double lobe structure having a banana shape, i.e. the distance between the lobes becomes larger from the center to the side parts.

Using existing OTR SPF models which are based on axial symmetry in the observation geometry the banana-shaped beam images could not be explained, and instead numerical simulations were carried out based on commercial ray tracing software [22]. Such numerical simulations are a powerful tool but may conceal the underlying physical processes. Thus, in our opinion a dedicated model which is directly adapted to the problem is better suited to achieve a closer insight and to develop strategies to cope with imperfections.

In this paper we present a simple approach to calculate two-dimensional OTR SPF dominated beam images which allows one to take into account the observation geometry, optical properties of the target under consideration as characterized by the Fresnel reflection coefficients, and partial imperfections in the optical system. Furthermore, a new fit function for SPF dominated beam imaging is proposed which allows a direct and fast beam size determination, thus paving the way for application of the method in online emittance diagnostics with submicron electron beams.

\section{THEORETICAL MODEL}

The problem is formulated in the following way: an electron that travels with the velocity $v=\beta c$ ( $c$ is the speed of light) interacts with a target (defined by the Fresnel reflection coefficients $\left|R_{\sigma}\right|^{2}$ and $\left|R_{\pi}\right|^{2}$ ) and generates backward emitted OTR, see Fig. 1. For the sake of simplicity it is assumed that the electron is ultrarelativistic, i.e. the condition $\gamma \gg 1$ holds for the Lorentz factor. This assumption allows one to neglect the influence of the longitudinal electric field component of the electron. The radiation is collected by an ideal thin lens having a focal length $f$ which generates an OTR based image in the image plane where a spatial resolving detector (CCD) is located. The action of the lens is to introduce a quadratic phase shift in the transverse electric field components [23]. In the subsequent consideration the influence of the prewave zone effect on the SPF is not taken into account because it was shown in Ref. [15] that it is negligible for realistic observation geometries even for $20 \mathrm{GeV}$ electrons.

In the subsequent brief theoretical outline the notations are adapted from Ref. [15]; for a more detailed treatment

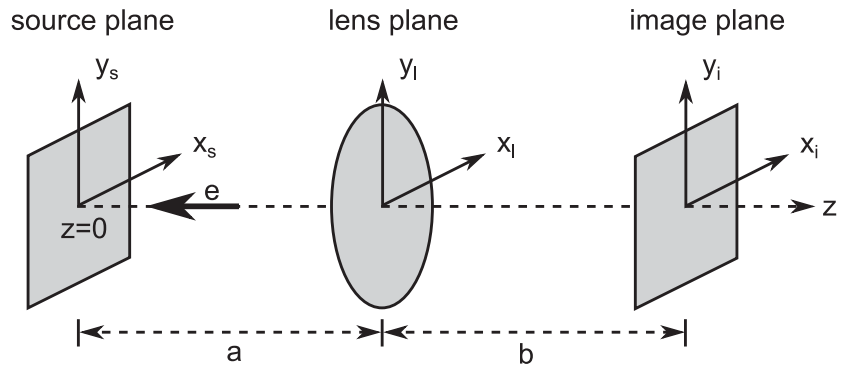

FIG. 1. Sketch of the calculation geometry. 
the reader is referred to this reference. The source field (electron field) can be represented in the form

$$
\begin{aligned}
E_{x_{s}, y_{s}}^{s}(\vec{r}, \omega) & =\frac{e \alpha}{\pi v} e^{-i \frac{\omega_{v} z}{v}} \frac{x_{s}, y_{s}}{\sqrt{x_{s}^{2}+y_{s}^{2}}} \mathrm{~K}_{1}\left(\alpha \sqrt{x_{s}^{2}+y_{s}^{2}}\right), \\
\text { with } \quad \alpha & =\frac{\omega}{\gamma v}
\end{aligned}
$$

which can be found in many textbooks, see e.g. Ref. [24]. Here $e$ is the electron charge, $\omega=\frac{2 \pi c}{\lambda}$, and $K_{1}$ is the modified Bessel function of the second kind. The pseudophoton transverse distribution described by Eq. (2) has a radial extension with a characteristic range of about $\gamma \lambda$, i.e. even in the case of a single electron the radiation source area has a finite radius which expands with increasing beam energy and wavelength of observation.

In the following, this source field is propagated through the optical system. The propagation is treated in the framework of scalar diffraction theory in the Fresnel approximation, i.e. up to the quadratic phase term. With the target located at $z=0$, the propagation from the source plane to the lens input situated at a distance $a$ away is described by

$$
\begin{aligned}
E_{x_{l}, y_{l}}^{l_{i}}\left(\vec{r}_{l}, \omega\right)= & -i \frac{e^{i k a}}{\lambda a} e^{i \frac{k}{2 a}\left(x_{l}^{2}+y_{l}^{2}\right)} \\
& \times \int_{\Sigma_{s}} \mathrm{~d}^{2} \Sigma_{s} E_{x_{s}, y_{s}}^{s}\left(\vec{r}_{s}, \omega\right) e^{i \frac{k}{2 a}\left(x_{s}^{2}+y_{s}^{2}\right)} e^{-i k^{\frac{x_{s} x_{l}+y_{s} y_{l}}{a}},}
\end{aligned}
$$

see for example Ref. [23]. The subscript $s$ indicates coordinates in the source and $l$ those in the lens plane, e.g. the source plane over which the integration carried out is denoted by $\Sigma_{s}$.

In the next step the resulting field Eq. (3) is propagated through the lens. The fields at the lens output are given by

$$
E_{x_{l}, y_{l}}^{l_{o}}\left(\vec{r}_{l}, \omega\right)=E_{x_{l}, y_{l}}^{l_{i}}\left(\vec{r}_{l}, \omega\right) e^{-i \frac{k}{2 f}\left(x_{l}^{2}+y_{l}^{2}\right)}
$$

where $f$ is the focal length of the lens.

Finally the field Eq. (4) is propagated from the lens output to the image plane which is situated at a distance $b$, see Fig. 1. This propagation is analogous to Eq. (3), and the result reads

$$
\begin{aligned}
E_{x_{i}, y_{i}}^{i}\left(\vec{r}_{i}, \omega\right)= & -i \frac{e^{i k b}}{\lambda b} e^{i \frac{k}{2 b}\left(x_{i}^{2}+y_{i}^{2}\right)} \\
& \times \int_{\Sigma_{l}} \mathrm{~d}^{2} \Sigma_{l} E_{x_{l}, y_{l}}^{l_{o}}\left(\vec{r}_{l}, \omega\right) e^{i \frac{k}{2 b}\left(x_{l}^{2}+y_{l}^{2}\right)} e^{-i k^{\frac{x_{l} x_{i}+y_{l} y_{i}}{b}}}
\end{aligned}
$$

with the integration performed over the lens surface. The subscript $i$ indicates coordinates in the image plane. The combination of Eqs. (3)-(5) results in the representation of the field distribution in the image plane as a function of the source and lens properties:

$$
\begin{aligned}
E_{x_{i}, y_{i}}^{i}\left(\vec{r}_{i}, \omega\right)= & -\frac{e \omega}{\pi \lambda^{2} \gamma a b \beta^{2} c^{2}} \int_{\Sigma_{l}} d^{2} \Sigma_{l} \\
& \times \exp \left(-i \frac{k\left(x_{l} x_{i}+y_{l} y_{i}\right)}{b}\right) \\
& \times \exp \left[-i \frac{k}{2}\left(x_{l}^{2}+y_{l}^{2}\right)\left(\frac{1}{f}-\frac{1}{a}-\frac{1}{b}\right)\right] \\
& \times \int_{\Sigma_{s}} d^{2} \Sigma_{s} \frac{x_{s}, y_{s}}{\sqrt{x_{s}^{2}+y_{s}^{2}}} K_{1}\left(\alpha \sqrt{x_{s}^{2}+y_{s}^{2}}\right) \\
& \times \exp \left(i \frac{k\left(x_{s}^{2}+y_{s}^{2}\right)}{2 a}\right) \\
& \times \exp \left(-i \frac{k\left(x_{s} x_{l}+y_{s} y_{l}\right)}{a}\right) .
\end{aligned}
$$

For the sake of simplicity the complex phase terms in front of the integrals are omitted because they do not contribute to the OTR intensity in the image plane which is the parameter accessible in the measurement and given by

$$
\frac{\mathrm{d}^{2} W}{\mathrm{~d} \omega \mathrm{d} \Omega}=\frac{c}{4 \pi^{2}}\left(\left|R_{\pi}\right|^{2}\left|E_{x_{i}}^{i}\right|^{2}+\left|R_{\sigma}\right|^{2}\left|E_{y_{i}}^{i}\right|^{2}\right)
$$

The source plane integration in Eq. (6) can be carried out analytically in polar coordinates as shown in Refs. [11-15]. The electric field in the image plane can be written as

$$
\begin{aligned}
E_{x_{i}, y_{i}}^{i}\left(x_{i}, y_{i}, \lambda\right)= & \frac{2 e}{\lambda M \beta c} \frac{\left\{x_{i}, y_{i}\right\}}{\sqrt{x_{i}^{2}+y_{i}^{2}}} \\
& \times \int_{0}^{\theta_{m}} d \theta \frac{\theta^{2}}{\theta^{2}+(\beta \gamma)^{-2}} J_{1}\left(\frac{2 \pi \sqrt{x_{i}^{2}+y_{i}^{2}} \theta}{\lambda M}\right) \\
& \times \exp \left[-i \frac{\pi}{\lambda} a^{2} \theta^{2}\left(\frac{1}{f}-\frac{1}{a}-\frac{1}{b}\right)\right] .
\end{aligned}
$$

Here $M=b / a$ is the magnification factor of the optical system in the case when $\frac{1}{f}=\frac{1}{a}+\frac{1}{b}, \theta=\frac{\sqrt{x_{l}^{2}+y_{l}^{2}}}{a}$ is the polar angle, and $J_{1}$ is the Bessel function of the first kind.

The authors in Refs. [11-14] assumed ideal focusing conditions, i.e. $f^{-1}=a^{-1}+b^{-1}$. As a result, the exponential phase factor in the integral in Eq. (8) disappears and the expression for the electric field can be further simplified.

In Ref. [15] however a small misalignment was introduced, i.e. $f^{-1}=(a+\Delta a)^{-1}+(b+\Delta b)^{-1}$, and it was demonstrated that this misalignment results in a broadening of the SPF even when $\Delta a / a \ll 1, \Delta b / b \ll 1$. Following this approach in the subsequent discussion it is assumed that $\Delta a, \Delta b \neq 0$, and the exponential phase factor in Eq. (8) can be rewritten in the general form

$$
-i \frac{\pi}{\lambda}\left(a_{0}+\Delta a\right)^{2} \theta^{2}\left(\frac{1}{f_{0}+\Delta f(\lambda)}-\frac{1}{a_{0}+\Delta a}-\frac{1}{b_{0}+\Delta b}\right)
$$




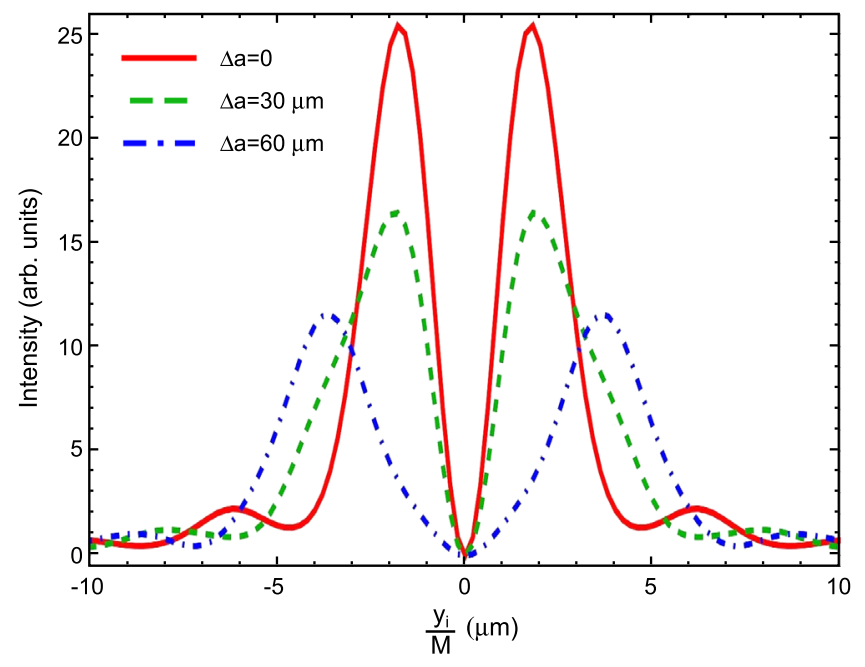

FIG. 2. Calculated OTR SPFs according to Eqs. (7)-(9). The parameter set for the calculation is indicated in the text.

with $\Delta f(\lambda)$ taking into account any existing chromatic aberration in the lens. The subscript 0 indicates ideal observation conditions, i.e. $f_{0}^{-1}=a_{0}^{-1}+b_{0}^{-1}$. In this situation the integral expression in Eq. (8) cannot be further simplified and has to be evaluated numerically.

In Fig. 2 one-dimensional OTR SPFs are shown which were calculated according to Eqs. (7)-(9). The parameter set used for the calculation and which will be used throughout this paper is $\gamma=1673, L=a+b=1183 \mathrm{~mm}$, $M=b / a=41.95, \lambda=400 \mathrm{~nm}, \theta_{m}=0.10,\left|R_{\sigma}\right|^{2}=1$ and $\left|R_{\pi}\right|^{2}=0$. A misalignment was assumed only in the object distance, and the misalignment parameters $\Delta a$ are stated in the figure.

As can be seen in Fig. 2 an increase of the misalignment parameter $\Delta a$ results in a significant deformation of the OTR SPF. Experimentally such a broadening was already observed for two different wavelengths $(\lambda=20 \mathrm{~nm}$ and $\lambda=400 \mathrm{~nm}$ ) in Ref. [17]; the parameter set used for these calculations corresponds to those experimental parameters. In the following only a misalignment $\Delta a$ is considered, however a similar broadening and deformation of the SPF will be observed in the case of chromatic lens aberrations.

\section{BEAM IMAGING IN THE CASE OF INCLINED TARGET}

To the authors' knowledge, with the exception of one design reported in Ref. [25] OTR is exclusively measured in the backward direction, i.e. the target acts like a mirror and the radiation appears in the vicinity of the specular angle. Typically, the angle $\Psi$ between target normal and electron trajectory is set at $45^{\circ}$ and the detector is placed under $90^{\circ}$ with respect to the beam axis. In this experimental geometry, the effect of the distortion of the transition radiation angular distribution caused by the influence of the electron's longitudinal electric field component becomes negligible for $\gamma>100$, cf. Ref. [26]. Nevertheless, smaller inclination angles are also in use in order to reduce the influence of depth of field [27]. In Refs. [28,29] it was demonstrated that the angular characteristics of backward transition and diffraction radiation of a single ultrarelativistic particle $(\gamma \gg 1)$ in the wave zone are determined with respect to the mirror reflection direction as long as the target inclination angle $\Psi$ satisfies the condition $\Psi \gg \gamma^{-1}$.

The approach developed in the previous section allows one not only to calculate the image of a single electron (i.e. the SPF), but also to determine the image of the whole beam under more realistic conditions. In this case the beam image is the sum of the individual SPFs generated by each electron inside the bunch. In previous publications, the authors calculated OTR based beam images under the assumption that all electrons inside a bunch have the same SPF [11-15]. In this case, the beam image is given by a convolution of the SPF with the transverse beam distribution. However, this approach is a simplification because the electrons inside the bunch generate different SPFs. This is especially the case for an inclined OTR target where the distances between the points of radiation generation on the target surface and the lens plane (i.e. the object distances) depend on the transverse electron position inside the bunch. Thus, in order to calculate the beam image taking into account the target tilt it is necessary to sum up the SPFs from the electrons inside the bunch.

In the following it is assumed that the target inclination corresponds to a rotation of the source plane around the $y_{s^{-}}$ axis. At first glance the target rotation results in a calculation difficulty because the axial symmetry with respect to the beam axis is broken, and the integration over the source plane in Eq. (6) should be performed in Cartesian coordinates which takes significant computational resources. Nevertheless, due to the fact that the OTR properties in the ultrarelativistic case do not depend specifically on the angle $\Psi$ it is still possible to use Eq. (8) for this calculation as illustrated in Fig. 3. In order to calculate the SPF from the $j$ th electron (indicated as $e_{j}^{-}$in Fig. 3), it is possible to treat the equivalent axial-symmetric problem assuming that the $j$ th electron generates a defocused $\mathrm{SPF}$, i.e. there is a misalignment in the object distance and $\Delta a_{j} \neq 0$.

Thus, the calculation of the beam image from an inclined target requires the determination of a series of twodimensional SPF images, taking into account that each emission point shifted horizontally along the target by $\Delta x_{e}^{j}$ has a different SPF. This SPF difference is a result of the position-dependent defocusing $\Delta a_{j}=\Delta x_{e}^{j} \tan \Psi$. Furthermore, it is necessary to take into account the geometrical shift of each $j$ th SPF in the image plane with respect to the central one by $\Delta x_{i}^{j}=\Delta x_{e}^{j}$. For electrons with a vertical offset $\Delta y_{e}^{j}$ in the source plane, all SPFs are assumed to be identical (because of no target tilt in this plane and therefore 


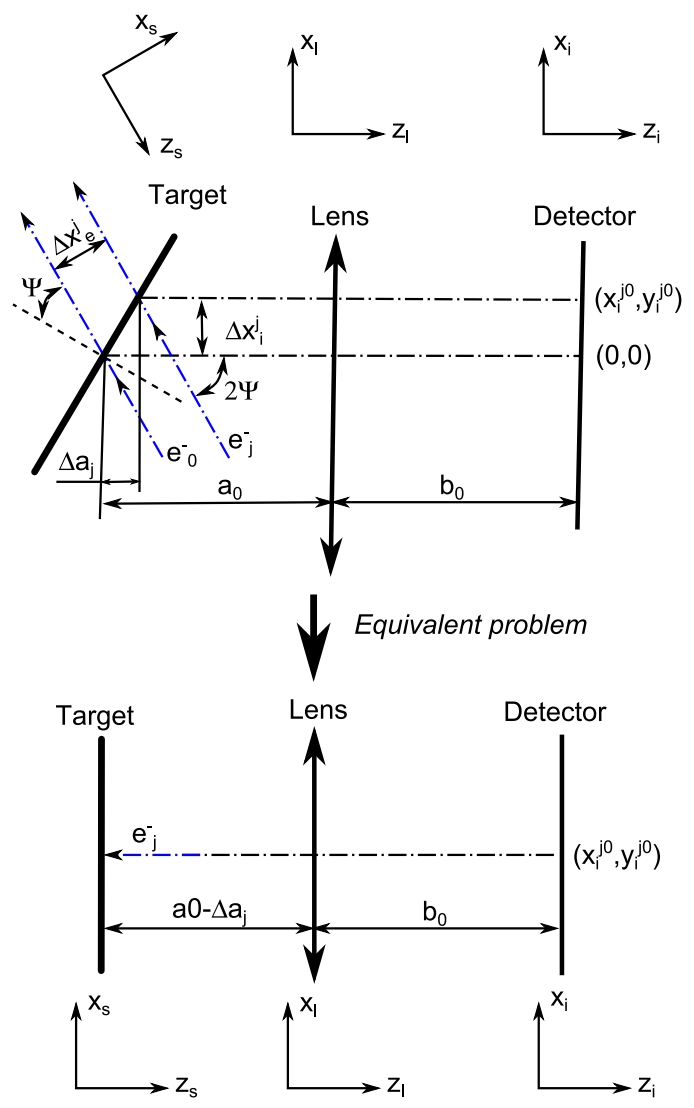

FIG. 3. Calculation scheme for the inclined target geometry together with geometrical definitions.

no defocusing) but shifted in the image plane by the same amount, i.e. $\Delta y_{i}^{j}=\Delta y_{e}^{j}$. In order to generate a beam image, all individual SPFs have to be summed up with a weight factor as defined by the transverse beam distribution. In the following, all calculations are carried out for two-dimensional Gaussian beam profiles which are characterized by their horizontal and vertical rms sizes $\sigma_{x}$ and $\sigma_{y}$, respectively.

For illustration purposes, Fig. 4 shows a set of calculated SPFs. In accordance with Fig. 3 it is assumed that the target is tilted around the vertical axis by $\Psi=45^{\circ}$, and the radiation emerges from horizontally displaced points along the tilted target surface, thus causing a misalignment which is characterized by the misalignment parameter $\Delta a=$ $\Delta x \tan \Psi$. The intensity weight factor is chosen such that

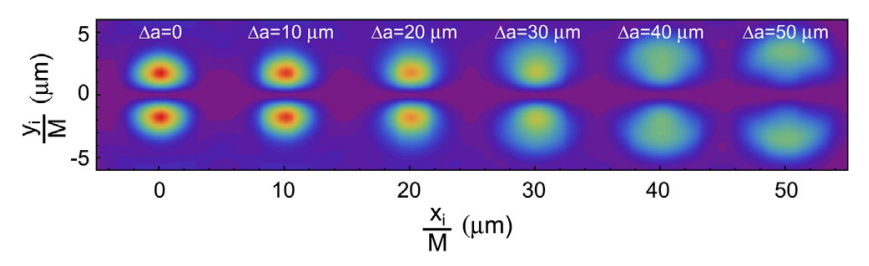

FIG. 4. SPFs calculated for horizontally displaced radiation emitters from a tilted target surface, emulating OTR emission from individual electrons out of a $\sigma_{x}=30 \mu \mathrm{m}$ thick beam profile. The numerical aperture amounts to $\theta_{m}=0.10$.

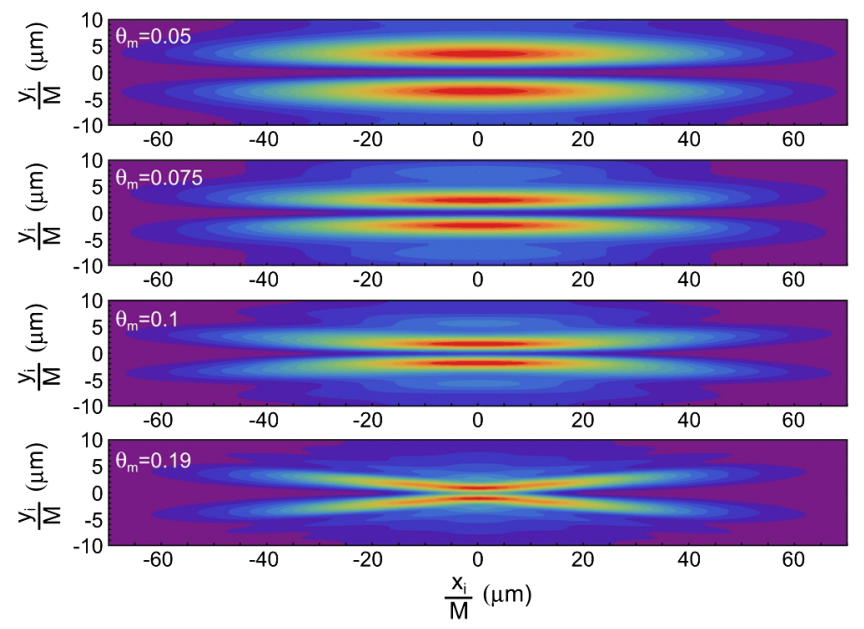

FIG. 5. Calculated two-dimensional beam images for a beam size of $\sigma_{x}=30 \mu \mathrm{m}, \sigma_{y}=0.4 \mu \mathrm{m}$ and for different numerical apertures $\theta_{m}$, in top-to-bottom order $\theta_{m}=0.05, \theta_{m}=0.075$, $\theta_{m}=0.10$, and $\theta_{m}=0.19$.

the SPFs shown in Fig. 4 correspond to OTR emission from selected electrons out of an electron bunch with $\sigma_{x}=30 \mu \mathrm{m}$ (rms) beam size. As can be seen from this illustration, the shape of the SPFs degrades with increasing misalignment parameter.

In Fig. 5 two-dimensional beam images are shown, which were calculated according to the inclined target model for an angle of $\Psi=45^{\circ}$, horizontal and vertical rms beam sizes of $\sigma_{x}=30 \mu \mathrm{m}$, respectively $\sigma_{y}=0.4 \mu \mathrm{m}$, lens apertures $\theta_{m}$ as indicated in the figure, and the same observation geometry as above. As can be seen from these results, an increase in the lens aperture $\theta_{m}$ results in a decrease of the interpeak distance in the distribution center as it is expected from the basic SPF theory. At the same time, the deformation of the image increases and shows a banana-like shape. This effect is clearly seen for the largest value of $\theta_{m}=0.19$. In this case, the peaks in the central part of the beam image are close together while at the sides the interpeak distance is much larger. As mentioned before, such banana-like beam images were observed by Kruchinin et al. in Ref. [21] for a target tilt angle of $\Psi=45^{\circ}$. This "banana-shape" beam image degradation is caused by the depth of field effect which is more pronounced for larger numerical lens apertures, resulting in a stronger influence of defocusing in the tilted (horizontal) plane with increasing distance from the rotation (y) axis.

For better illustration, Fig. 6 shows examples of vertical cross sections taken from the beam images in Fig. 5 for two distinct horizontal positions: at $x_{i}=0$ where the focal condition is satisfied and at $x_{i} / M=40 \mu \mathrm{m}$ which is out of focus. As can be seen from this figure, while taking into account finite beam sizes the central minimum is smeared out compared to the bare SPFs in Fig. 2, and this effect is more pronounced as the numerical aperture of the lens increases. Furthermore, in the central part of the beam 


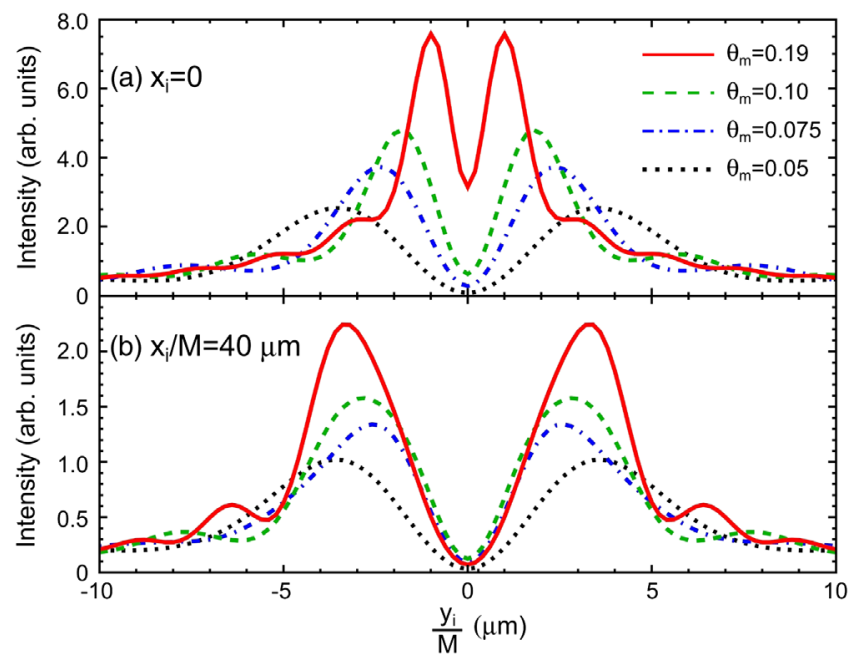

FIG. 6. Examples of vertical beam image cross sections, taken from Fig. 5 at two different horizontal positions (a) $x_{i}=0$ and (b) $x_{i} / M=40 \mu \mathrm{m}$ with the numerical aperture $\theta_{m}$ as parameter: $\theta_{m}=0.19$ (red solid line), $\theta_{m}=0.10$ (green dashed line), $\theta_{m}=$ 0.075 (blue dash-dotted line), and $\theta_{m}=0.05$ (black dotted line).

image $\left(x_{i}=0\right)$ the minimum smears out much more with increasing $\theta_{m}$ compared to the side part of the beam image at $x_{i} / M=40 \mu \mathrm{m}$ due to the smaller interpeak distance.

As can be seen from these results, a measurement of an SPF dominated beam image is very sensitive to beam size effects, and the sensitivity of this method is higher for a large numerical aperture of the optical system and for the central part of the image where the imaging condition is satisfied. However, information about the beam size can still be obtained even if the outer parts of the image are out of focus; this will be discussed in the next section. For the analysis of SPF dominated images for the measurement of small vertical beam sizes, SPF dominated Gaussian beam images can be divided into three cases.

In the first case the rms beam size is smaller than the position of the maxima in the SPF distribution, an example is given in Fig. 6(a) for $\theta_{m}=0.19$. The increase of the beam size results in a smearing out of the central minimum. In this case the beam size can be extracted via the ratio of the maximum and the central minimum intensities. This ratio is usually called the visibility $V$ and is defined as

$$
V=\frac{I_{\max }-I_{\min }}{I_{\max }+I_{\min }}
$$

with $I_{\max }$ the radiation intensity at the maximum and $I_{\min }$ at the central minimum. In the case of nonzero visibility it is possible to apply a special fit function that fits the experimental data and helps to extract the visibility. Such a fit function was presented by Aryshev et al. [20] and by Kruchinin et al. [21]. However, it should be emphasized that the fit function proposed in Refs. [20,21] allows one to obtain only the visibility of the beam image, but not the absolute value of the beam size. In order to extract the beam size, a special procedure was developed in Ref. [20] which is based on a self-calibration of the optical scheme, resulting in a functional dependence of the rms beam size on the visibility. In order to carry out this self-calibration, a set of SPF dominated beam images is required for different beam sizes, and an important prerequisite is the presence of a beam image with high visibility. As a consequence, this method requires sufficient flexibility in beam size generation, and cannot be applied for a single SPF dominated beam image.

In the second case the rms beam size is about a factor of 10 larger than the SPF maxima position. In this case, the SPF influence can be neglected and the beam size can simply be extracted using a standard fit procedure with a Gaussian fit function.

The third case is when the beam size is in an intermediate region, i.e. the optical system images the Gaussian beam in such a way that it has neither a pure Gaussian nor a double lobe distribution. The authors in Ref. [22] call this region a "blind area" where a beam size determination does not seem to be possible with any of the existing approaches.

In order to circumvent the difficulties with the selfcalibration in the first case and to allow even beam size determination in the third case, a new approach has been developed and will be presented in the next section.

\section{FIT FUNCTION FOR THE ANALYSIS OF SPF DOMINATED IMAGES}

In this section a fit function is proposed by which it is possible to extract beam size information for the SPFdominated and the blind regions (first and third cases above). It is assumed that a flat Gaussian beam is to be analyzed with the horizontal beam size $\sigma_{x}$ being much larger than the vertical one $\sigma_{y}$. In this situation, the problem of a twodimensional beam size analysis is reduced to the determination of a Gaussian distributed horizontal beam profile (second case above) which can be solved using standard fit methods, and the determination of SPF-dominated vertical beam profiles. Furthermore, it is assumed that (i) only the $\sigma$-polarization component is detected which can be realized experimentally either by a proper choice of the reflection geometry or by using polarization filters, and that (ii) beam imaging is performed with monochromatic radiation.

The problem of fitting an SPF dominated beam profile will be divided into two parts. The first part is to find a simple analytical function that can be used to approximate the bare SPF in the region of interest where the sensitivity on the vertical beam size is highest. The second part is to adopt this function for fitting SPF dominated beam images, i.e. images which are composed of an SPF convolution with a Gaussian beam distribution, even showing influence of defocusing.

The following function is well suited for a fit of the central part of the SPF where the focusing condition holds, i.e. at $x_{i}=0$ 


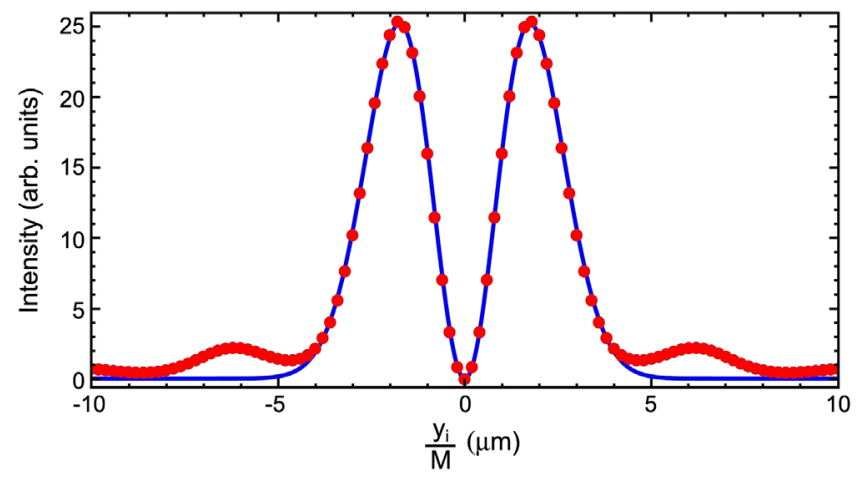

FIG. 7. Calculated vertical SPF profile (points) and fit (solid line) according to Eq. (11). The SPF calculation parameters are those of Fig. 2 for ideal focusing $(\Delta a=0)$.

$$
f_{1}\left(y_{i}\right)=q_{0} y_{i}^{2} \exp \left[-q_{1}^{-2} y_{i}^{2}\right]
$$

Here $q_{0}$ and $q_{1}$ are free fit parameters: $q_{0}$ describes an amplitude factor and $q_{1}$ directly represents the peak position of the SPF spatial distribution.

As an example, Fig. 7 shows a calculated SPF distribution together with a fit according to Eq. (11). In this case, the SPF plotted in Fig. 2 for ideal focusing $(\Delta a=0)$ was used, and the values of the resulting fit parameters and their standard errors are $q_{0}=22.6 \pm 0.25$ and $q_{1}=$ $(1.74 \pm 0.01) \mu \mathrm{m}$. The fit procedure was carried out with the Wolfram Mathematica software, using the built-in function NonlinearModelFit which is a least-squares fit routine based on the Levenberg-Marquardt algorithm. Each point of the SPF distribution was weighted with a weight factor proportional to the square root of the intensity. As can be seen in Fig. 7, the fit function Eq. (11) approximates well the central part of the SPF distribution, while the outer parts in the region of higher order maxima and minima are not well described.

The important feature of the fit function $f_{1}$ Eq. (11) is that the convolution with a Gaussian beam distribution can be expressed analytically, resulting in

$$
\begin{aligned}
f_{2}\left(y_{i}\right) & =\int_{-\infty}^{\infty} \frac{1}{\sqrt{2 \pi} q_{2}} \exp \left[-\frac{y_{0}^{2}}{2 q_{2}^{2}}\right] f_{1}\left(y_{i}-y_{0}\right) d y_{0} \\
& =\frac{q_{0} q_{1}^{2}}{q_{2}} \frac{1}{\sqrt{2 q_{1}^{-2}+q_{2}^{-2}}} \frac{2 q_{2}^{4}+q_{1}^{2}\left(q_{2}^{2}+y_{i}^{2}\right)}{\left(q_{1}^{2}+2 q_{2}^{2}\right)^{2}} e^{-\frac{y_{i}^{2}}{q_{1}^{2}+2 q_{2}^{2}}}
\end{aligned}
$$

with $q_{2}$ an additional free fit parameter which describes the vertical $\mathrm{rms}$ beam size. In the following the rms vertical beam size obtained from the fit functions will be denoted as $\sigma_{y}^{\mathrm{fit}} \equiv q_{2}$. For the data fitting procedure the amplitude factor $q_{0} q_{1}^{2} / q_{2}$ in Eq. (12) which consists of three independent fit parameters was combined to a common scaling factor $Q_{0}$ which was solely used for data scaling.

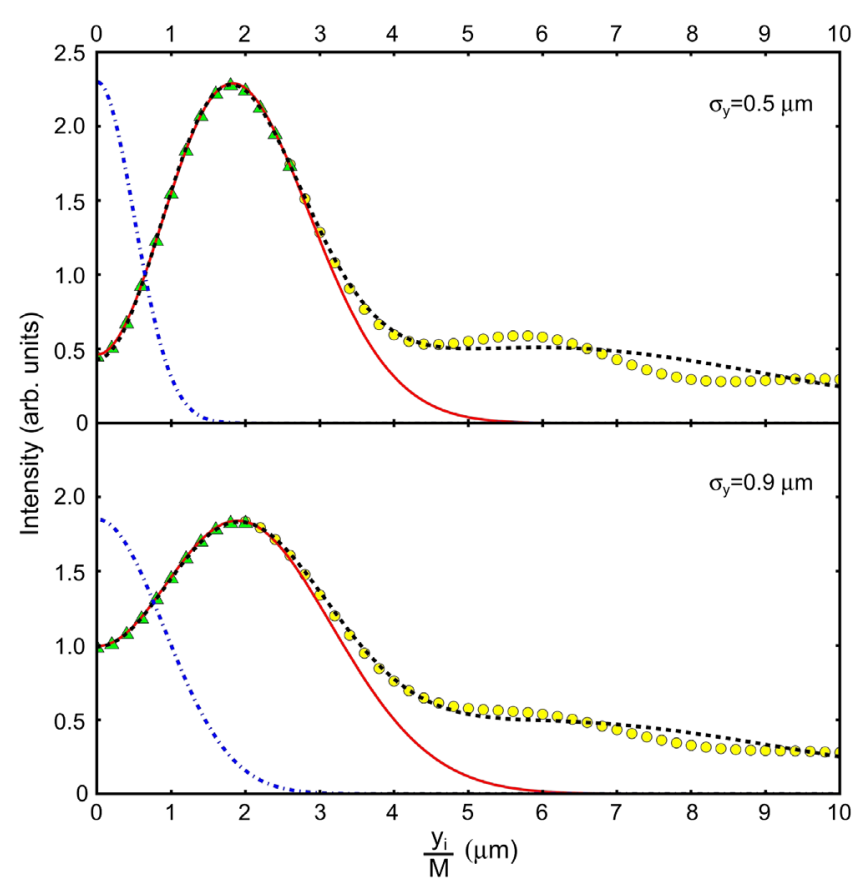

FIG. 8. Calculated vertical beam profiles at $x_{i}=0$ (green triangles and yellow points) together with the fitted functions according to Eq. (12) (red solid line) and to Eq. (13) (black dashed line). The vertical beam profile is indicated by the blue dash-dotted line. The beam image calculation parameters are the same as in Fig. 5 for $\theta_{m}=0.1$.

For illustration Fig. 8 shows quality studies of the fit function Eq. (12) based on calculated beam images and assuming two slightly different beam sizes of $\sigma_{y}=0.5 \mu \mathrm{m}$ and $\sigma_{y}=0.9 \mu \mathrm{m}$ together with the beam profiles (blue dash-dotted line). Because of the beam image symmetry only half of the vertical profiles are plotted. In this figure the yellow dots and green triangles correspond to the calculated profile values at $x_{i}=0$ and for $\theta_{m}=0.1$, the different colors and symbols indicate two different ranges for the fit analysis. The red solid line corresponds to the fit curve according to Eq. (12) which was applied only to the part of the calculated profile marked by the green triangles. This range comprises 27 points for $\sigma_{y}=0.5 \mu \mathrm{m}$ and 21 points for $\sigma_{y}=0.9 \mu \mathrm{m}$. For the beam size of $\sigma_{y}=0.5 \mu \mathrm{m}$ the extracted fit parameter values are $q_{1}=(1.77 \pm$ $0.001) \mu \mathrm{m}$ and $\sigma_{y}^{\mathrm{fit}}=(0.502 \pm 0.001) \mu \mathrm{m}$, for the size of $\sigma_{y}=0.9 \mu \mathrm{m}$ the parameter values are $q_{1}=(1.78 \pm$ $0.001) \mu \mathrm{m}$ and $\sigma_{y}^{\mathrm{fit}}=(0.906 \pm 0.001) \mu \mathrm{m}$. As can be seen from this comparison, there is a rather good agreement between the input beam sizes used for the calculation and the fitted ones. However, the fit works well only for the central part of the beam image profile due to the fact that the tails of the SPF distribution are not taken into account by the initial function Eq. (11). An increase in the number of data points for the fit range would result in inaccurate fit results due to the increase in the distribution tail influence. 
The authors are aware of the difficulty in beam size determination using the fit function Eq. (12) because only the central part can be exploited for the analysis. The problem is that it is not clear beforehand how far this central part will extend. This problem can be overcome by performing a convergence analysis, i.e. by applying the fit procedure and determining the beam size as a function of the extension of the central region. According to the authors' experience such analysis can straightforward be performed in order to extract trustworthy beam size values.

Apart from that it is possible to modify the fit function Eq. (12) such that it can be applied to a larger range, thus taking into account also the distribution tails. Having in mind the advantage of an analytical expression for the convolution with a Gaussian beam distribution, the fit functions can be modified as follows:

$$
\begin{aligned}
f_{1}^{\bmod }\left(y_{i}\right)= & q_{0} y_{i}^{2}\left(\exp \left[-q_{1}^{-2} y_{i}^{2}\right]+q_{3} \exp \left[-\left(q_{4} q_{1}\right)^{-2} y_{i}^{2}\right]\right) \\
f_{3}\left(y_{i}\right)= & \int_{-\infty}^{\infty} \frac{1}{\sqrt{2 \pi} q_{2}} \exp \left[-\frac{y_{0}^{2}}{2 q_{2}^{2}}\right] f_{1}^{\bmod }\left(y_{i}-y_{0}\right) d y_{0} \\
= & Q_{0}\left(\frac{1}{\sqrt{2 q_{1}^{-2}+q_{2}^{-2}}} \frac{2 q_{2}^{4}+q_{1}^{2}\left(q_{2}^{2}+y_{i}^{2}\right)}{\left(q_{1}^{2}+2 q_{2}^{2}\right)^{2}} e^{-\frac{y_{i}^{2}}{q_{1}^{2}+2 q_{2}^{2}}}\right. \\
& +\frac{q_{3}}{\sqrt{2\left(q_{4} q_{1}\right)^{-2}+q_{2}^{-2}}} \\
& \left.\times \frac{2 q_{2}^{4}+\left(q_{4} q_{1}\right)^{2}\left(q_{2}^{2}+y_{i}^{2}\right)}{\left(\left(q_{4} q_{1}\right)^{2}+2 q_{2}^{2}\right)^{2}} e^{-\frac{y_{i}^{2}}{\left(q_{4} q_{1}\right)^{2}+2 q_{2}^{2}}}\right) .
\end{aligned}
$$

This change of the fit function results in the appearance of a second term that approximates the contribution of the side lobes. To achieve this, the range of the fit parameter $q_{3}$ should be limited to $q_{3} \in[0,1]$. To the authors' experience, with an initial value of $q_{3}^{\text {in }}=0.1$ the fit converged fast and successfully all the time. Furthermore, $q_{4}$ is not a free fit parameter but has to be set based on the calculation of the SPF. It defines the position of the side lobes in the SPF distribution with respect to the position of the first (central) lobe. In the case of ideal focusing it was set to $q_{4}=3.5$. For better illustration Fig. 9 shows the SPF distribution of Fig. 7 on a logarithmic scale together with the fit based on function $f_{1}^{\bmod }\left(y_{i}\right)$ in Eq. (13), resulting in the free fit parameter values of $q_{0}=22.9 \pm 0.1, q_{1}=(1.72 \pm 0.003) \mu \mathrm{m}$, and $q_{3}=0.005 \pm$ 0.0002 . In addition, the individual contributions from the fit of the central lobe (blue solid line) and the side lobes (blue dashed line) are shown, indicating that the averaged side lobe contribution is described in a satisfactory way.

The modified fit function $f_{3}\left(y_{i}\right)$ in Eq. (13) was also tested using the calculated vertical beam image data shown in Fig. 8. In this case the fit was applied to the whole range of data marked by green triangles and yellow points together, and the result is indicated by the black dashed line in Fig. 8. As can be seen from this comparison, the average

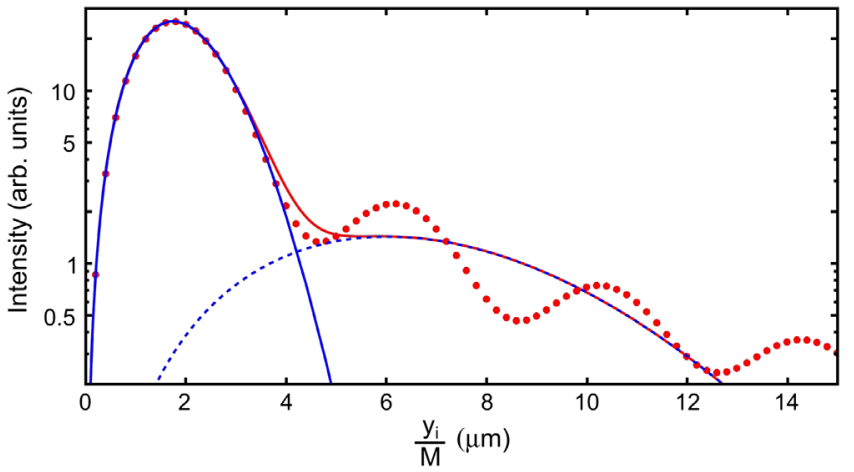

FIG. 9. SPF distribution of Fig. 7 plotted on a logarithmic scale (red circles) together with fit based on $f_{1}^{\bmod }\left(y_{i}\right)$ Eq. (13) (red line). In addition, the individual contributions from the fit of the central lobe (blue solid line) and the side lobes (blue dashed line) are shown.

contribution of the side lobes is well approximated by this fit. The extracted fit parameter values together with their standard errors are $q_{1}=(1.70 \pm 0.009) \mu \mathrm{m}, \sigma_{y}^{\mathrm{fit}}=$ $(0.475 \pm 0.014) \mu \mathrm{m}$ for the input beam size of $\sigma_{y}=0.5 \mu \mathrm{m}$, and $q_{1}=(1.72 \pm 0.013) \mu \mathrm{m}, \sigma_{y}^{\mathrm{fit}}=(0.9 \pm$ $0.013) \mu \mathrm{m}$ for $\sigma_{y}=0.9 \mu \mathrm{m}$. Comparing these values with those obtained for the fit function based on Eq. (12) one can see that the modified function gives reasonable results for the beam size $\sigma_{y}=0.5 \mu \mathrm{m}$ but better ones for $\sigma_{y}=0.9 \mu \mathrm{m}$. At the same time the whole distribution range is used, resulting in a much simpler analysis of the beam image.

One of the advantages of the proposed fit function Eq. (13) is the fact that it allows one to extract also beam sizes from SPF dominated beam images in the "blind zone" where the beam image has neither a double lobe structure nor a Gaussian distribution. For demonstration Fig. 10 shows a calculated beam image that was analyzed with the help of Eq. (13). The fit was performed for vertical cuts at different horizontal positions in the image $\left(x_{i}=0, x_{i} / M=10 \mu \mathrm{m}\right.$, $x_{i} / M=20 \mu \mathrm{m}$ ), and following Ref. [22] the profile from the central position corresponds to the situation of data analysis in the blind zone. Figure 10 shows the comparison for the SPF dominated beam image, calculated for $\theta_{m}=0.19$, $\lambda=400 \mathrm{~nm}$, and $\sigma_{y}=1 \mu \mathrm{m}$, together with the associated profile fits. All points of each profile were taken into account and all fitting parameters in Eq. (13) were assumed to be free ones except $q_{4}=3.5$. The fit value of the rms vertical beam size $\sigma_{y}^{\text {fit }}$ is taken and compared to the input beam size $\sigma_{y}=1 \mu \mathrm{m}$ used for the calculation. The values of the fitted beam sizes are quoted in the caption of Fig. 10.

As can be seen from this comparison, the central part of the calculated beam profiles is well described by the fit function. The extracted beam sizes are in reasonable agreement with the input beam size, the maximum deviation between fitted and input beam size is better than $16 \%$. Since information about the beam size can be gained not only from the central part, now it is possible to 

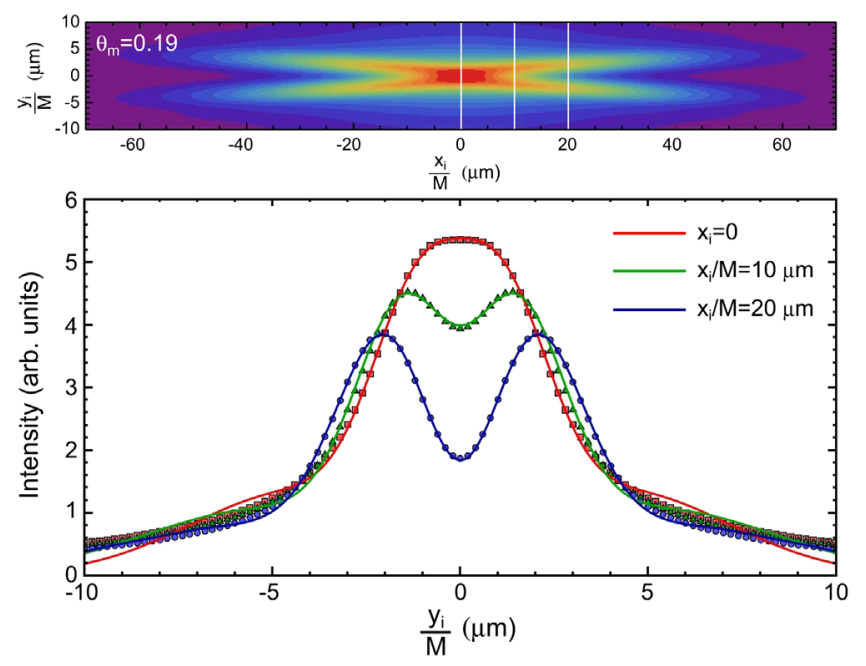

FIG. 10. Upper plot: Beam image calculated for $\theta_{m}=0.19$, $\lambda=400 \mathrm{~nm}, \sigma_{y}=1 \mu \mathrm{m}$. Lower plot: Comparison of vertical beam profiles taken at different horizontal positions with the fit of the central region according to Eq. (13). The points represent the calculated profiles, the solid lines the fitted curves. The horizontal positions are $x_{i}=0$ (red squares/line, $\sigma_{y}^{\text {fit }}=1.16 \pm 0.06 \mu \mathrm{m}$ ), $x_{i} / M=10 \mu \mathrm{m}$ (green triangles/line, $\sigma_{y}^{f i t}=1.08 \pm 0.02 \mu \mathrm{m}$ ), and $x_{i} / M=20 \mu \mathrm{m}$ (blue dots/line $\sigma_{y}^{f i t}=0.92 \pm 0.01 \mu \mathrm{m}$ ).

determine $\sigma_{y}$ in the cases of any artifacts in the central part of beam image. However, the authors should mention that the use of the side parts of the image is not a "standard regime" for the proposed fit function due to the fact that the SPF for the case of defocusing is wider (see Fig. 2) and therefore is not well described neither by the SPF fit function $f_{1}$ Eq. (11) nor by $f_{1}^{\text {mod }}$ Eq. (13).

Figure 11 shows a comparison of extracted beam sizes $\sigma_{y}^{\text {fit }}$ as a function of the input beam size for two different fit functions, the proposed function according to Eq. (13) and a standard Gaussian fit function. For this comparison a set of beam images was calculated for beam sizes in the range of $0.4 \mu \mathrm{m}$ up to $20 \mu \mathrm{m}$, using the parameter set $\theta_{m}=0.1$, $\lambda=400 \mathrm{~nm}, \sigma$-polarization, and the vertical profiles were generated at the central cut $x_{i}=0$. For all data sets the fit was performed in the same way, i.e. with the same initial values for the free running fit parameters. For simplification, the central lobe position $q_{1}$ in Eq. (13) was kept fix at $q_{1}=1.71 \mu \mathrm{m}$. This procedure is permitted because $q_{1}$ does not depend on the beam size but is defined in advance from the experimental parameters simply by calculating the SPF function. As can be seen from Fig. 11, the fit based on Eq. (13) shows a good agreement over the whole range of beam sizes. The gray dashed line highlights the beam size at which the visibility becomes equal to zero and therefore the transition from SPF-dominated imaging to the blind zone. At the same time, the fit based on the Gaussian function results in beam sizes having even larger deviations due to the SPF influence which is not taken into account in this description. For beam sizes larger than $\sigma_{y}=10 \mu \mathrm{m}$,

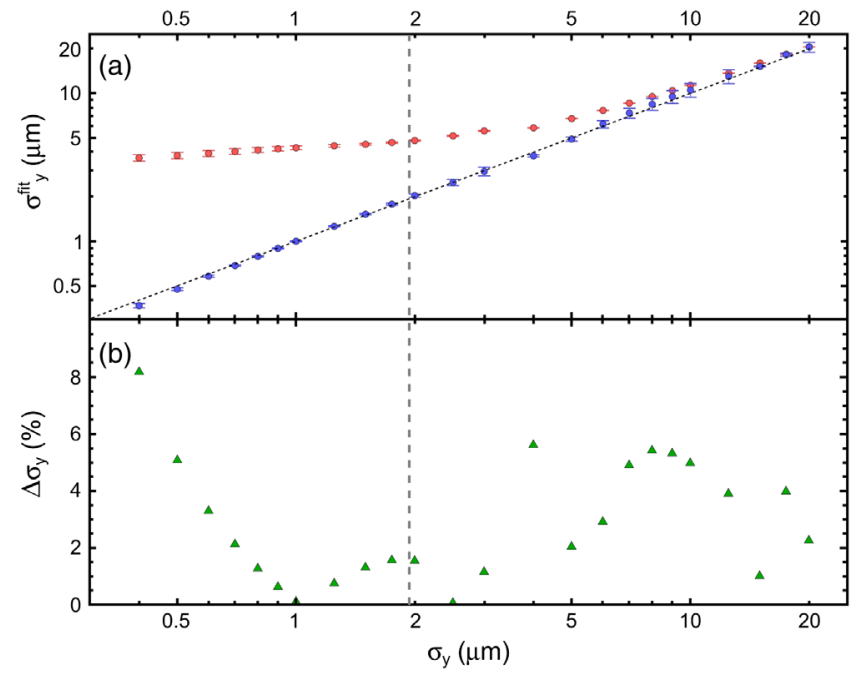

FIG. 11. (a) Comparison of two different fit functions applied to extract vertical beam sizes from calculated beam images. Parameter set for calculation: $\theta_{m}=0.1, \lambda=400 \mathrm{~nm}$, and OTR $\sigma$-polarization component. Red points: proposed fit function Eq. (13); blue points: Gaussian fit function. The black solid line indicates ideal coincidence between input and fitted beam sizes, the gray dashed line shows the beam size at which the visibility becomes equal to zero. (b) Relative difference between input and fitted beam sizes according to Eq. (13).

both fit functions result in similar $\sigma_{y}^{\text {fit }}$ values having a good agreement with the input size because the influence of the SPF on the beam profile becomes negligible. The discrepancy between the input beam sizes and the ones extracted from the fit procedure based on the proposed fit function Eq. (13) is smaller than $10 \%$ over the whole range as can be seen in Fig. 11(b).

The last point to be outlined is the behavior of the fit based on Eq. (13) under even more realistic conditions, i.e. when the input data do not follow a smooth theoretical curve but are noisy as it is the case in a real experiment. Therefore the beam profile data from Fig. 8 were taken for a

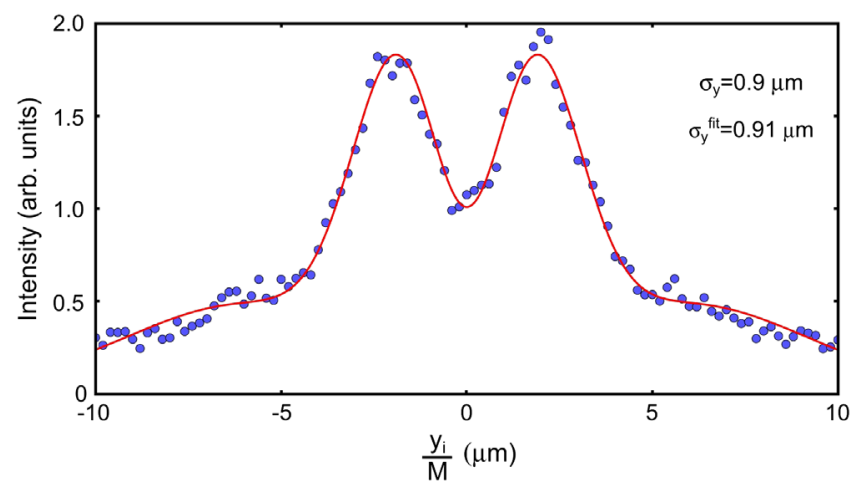

FIG. 12. Comparison of beam profile data (blue points) with fit function Eq. (13) (red solid line) under real experimental conditions. For the profile the data from Fig. 8 were taken for a beam size of $\sigma_{y}=0.9 \mu \mathrm{m}$ and a random noise was added. 
beam size of $\sigma_{y}=0.9 \mu \mathrm{m}$, and a random noise was added. The noise was normally distributed with rms value equal to $\mathrm{rms}_{i}^{\text {noise }}=0.1 I_{i}$, where $I_{i}$ is the intensity at the point $y_{i}$. Figure 12 shows the simulated data together with the fit. The extracted beam size value of $\sigma_{y}^{\mathrm{fit}}=(0.91 \pm 0.1) \mu \mathrm{m}$ is encouraging and gives confidence that it will be possible to extract accurate beam size information under real experimental conditions with submicrometer beam sizes. With the method proposed here, the complete beam size information can be gained from only one beam image, thus avoiding the inherent difficulties to produce data sets for self-calibration as it was proposed in Ref. [20].

\section{SUMMARY AND CONCLUSION}

This paper presents a simple theoretical model that allows one to calculate two-dimensional beam images from tilted targets using OTR in the visible spectral region. According to Eq. (9), the model allows one to take into account misalignments in the optical setup and chromatic aberration in the optical system, as well as the reflection coefficients of the target. With the help of this model it is possible to estimate the influence of the target tilt angle on the observed beam image.

Simulation results based on the model show the presence of the depth-of-field effect for tilted targets, causing an increase in the interpeak distance of SPF dominated beam images in regions which are out of focus (i.e. in regions far away from the optical axis). As is the case for conventional optical systems, the depth-of-field influence is more pronounced for large numerical lens apertures, see Fig. 5. Despite the fact that an increase of the lens numerical aperture increases the depth-of-field contribution, it additionally enhances the sensitivity to a change in small beam sizes, demonstrated in Fig. 6. Thus, based on the model and depending on the expected beam size range an optimization of the lens numerical aperture can be performed in order to balance both effects.

In addition, a new fit function Eq. (13) is proposed which can be used to extract vertical beam sizes from SPF dominated beam images. Advantages of this function are its simplicity, i.e. the free fit parameters have a clear physical meaning, and that it does not require to construct any calibration function in order to estimate the beam size as in the case in Ref. [20]. In addition, it has a wider range of applicability because it allows one to extract beam size information not only from images expressing a clear double lobe structure caused by the SPF, but also from the intermediate range (so-called blind area) where the beam image profile has neither a double-lobe nor a Gaussian structure.

In order to emphasize this advantage, extracted fit parameters from the proposed fit function are compared to the ones using a conventional Gaussian function for beam profile description over a wide range of vertical beam sizes which cover the three cases of SPF-dominated beam images, intermediate range (blind area), and the range of pure Gaussian images. The fit based on Eq. (13) is shown to be advantageous in the SPF-dominated and the intermediate range, and presents no disadvantages for the analysis of pure Gaussian beam images because of the negligible SPF influence. In order to increase the sensitivity of beam size determination in the blind area based on the new fit function, it is proposed to introduce a dedicated misalignment in the optical system which results in the degradation of the resolution such that the beam image shape will degrade to a double-lobe shape.

\section{ACKNOWLEDGMENTS}

We express our gratitude to M. Lomperski (DESY) and A. Aryshev (KEK) for fruitful discussions and careful proofreading of the manuscript. The work was partly supported by Russian Ministry of Education and Science within the program "Nauka", Grant No. 3.1903.2017. L. G. would also like to express gratitude to PIER (partnership between Hamburg University and DESY), fellowship PFS-2015-06 and Fellowship of the Russian Federation President (SP-2406.2016.2).

[1] R. B. Fiorito and D. W. Rule, Optical transition radiation beam emittance diagnostics, AIP Conf. Proc. 319, 21 (1994).

[2] L. Wartski, S. Roland, J. Lasalle, M. Bolore, and G. Filippi, Interference phenomenon in optical transition radiation and its application to particle beam diagnostics and multiplescattering measurements, J. Appl. Phys. 46, 3644 (1975).

[3] K. Honkavaara et al., Design of OTR beam profile monitors for the Tesla Test Facility, phase 2 (TTF2), in Proceedings of the Particle Accelerator Conference, PAC-03, Portland, Oregon, 2003 (IEEE, Piscataway, New Jersey, 2003), p. 2476.

[4] F. Löhl, S. Schreiber, M. Castellano, G. Di Pirro, L. Catani, A. Cianchi, and K. Honkavaara, Measurements of the transverse emittance at the FLASH injector at DESY, Phys. Rev. ST Accel. Beams 9, 092802 (2006).

[5] R. B. Fiorito, Recent advances in OTR beam diagnostics, in Proceedings of the Particle Accelerator Conference, PAC-09, Vancouver, Canada, 2009 (JACoW, Piscataway, New Jersey, 2009), TU3GRI02, p. 741.

[6] R. B. Fiorito, D. Feldman, A. G. Shkvarunets, S. Casey, B. L. Beaudoin, B. Quinn, and P. G. O'Shea, OTR measurements of the $10 \mathrm{keV}$ electron beam at the university of Maryland electron ring (UMER), in Proceedings of the Particle Accelerator Conference, PAC-07, Albuquerque, New Mexico, 2007 (IEEE, Piscataway, New Jersey, 2007), FRPMS03, p. 4006.

[7] P. Catravas, W. P. Leemans, E. Esarey, M. Zolotorev, D. Whittum, R. Iverson, M. Hogan, and D. Walz, Beam profile measurement at $30 \mathrm{GeV}$ using optical transition radiation, in Proceedings of the Particle Accelerator Conference, PAC-99, New York, 1999 (IEEE, Piscataway, New Jersey, 1999), p. 2111. 
[8] O. V. Afanasyev, A. B. Baluev, K. I. Gubrienko, E. A. Merker, K. Wittenburg, and I. Krouptchenkow, A beam shape oscillation monitor for HERA, AIP Conf. Proc. 868, 534 (2006).

[9] V. E. Scarpine, A. H. Lumpkin, and G. R. Tassotto, Initial OTR measurements of $150 \mathrm{GeV}$ protons in the tevatron at FNAL, AIP Conf. Proc. 868, 473 (2006).

[10] B. Walasek-Höhne, C. Andre, F. Becker, P. Forck, A. Reiter, M. Schwickert, and A. Lumpkin, Optical transition radiation for non-relativistic ion beams, in Proceedings of the High-Intensity and High-Brightness Hadron Beams, Beijing, China, 2012 (JACoW, Geneva, 2012), THO3C01, p. 580.

[11] V. A. Lebedev, Diffraction-limited resolution of the optical transition radiation monitor, Nucl. Instrum. Methods Phys. Res., Sect. A 372, 344 (1996).

[12] M. Castellano and V. A. Verzilov, Spatial resolution in optical transition radiation beam diagnostics, Phys. Rev. ST Accel. Beams 1, 062801 (1998).

[13] A. P. Potylitsyn, Image of optical diffraction radiation (ODR) source and spatial resolution of ODR beam profile monitor, in Advanced Radiation Sources and Applications, NATO Science Series II: Mathematics, Physics and Chemistry Vol. 199, edited by H. Wiedemann (Springer, Dordrecht, 2006), pp. 146-163.

[14] D. Xiang and W.-H. Huang, Theoretical considerations on imaging of micron size electron beam with optical transition radiation, Nucl. Instrum. Methods Phys. Res., Sect. A 570, 357 (2007).

[15] G. Kube, Report No. TESLA-FEL 2008-01, 2008.

[16] G. Stupakov, Report No. SLAC-PUB-14758, 2011.

[17] G. Kube, S. Bajt, Yu. A. Popov, A. P. Potylitsyn, L. G. Sukhikh, and W. Lauth, Transverse beam profile diagnostics using point spread function dominated imaging with dedicated de-focusing, in Proceedings of the International Particle Accelerator Conference, Shanghai, China, 2013 (JACoW, Shanghai, China, 2013), MOPME010, p. 488.

[18] L. G. Sukhikh, G. Kube, S. Bajt, W. Lauth, Yu. A. Popov, and A. P. Potylitsyn, Backward transition radiation in the extreme ultraviolet region as a tool for the transverse beam profile diagnostic, Phys. Rev. ST Accel. Beams 17, 112805 (2014).

[19] P. Karataev, A. Aryshev, S. Boogert, D. Howell, N. Terunuma, and J. Urakawa, First Observation of the Point Spread Function of Optical Transition Radiation, Phys. Rev. Lett. 107, 174801 (2011).
[20] A. Aryshev, N. Terunuma, J. Urakawa, S. T. Boogert, P. Karataev, and D. Howell, Sub-micrometer resolution transverse electron beam size measurement system based on optical transition radiation, in Proceedings of the 2011 International Particle Accelerator Conference, San Sebastian, Spain, 2011 (JACoW, New York, 2011), WEOBB01, p. 1965.

[21] K. Kruchinin, S. T. Boogert, P. Karataev, L. J. Nevay, B. Bolzon, T. Lefevre, S. Mazzoni, A. Aryshev, M. Shevelev, N. Terunuma, and J. Urakawa, Extremely low emittance beam size diagnostics with sub-micrometer resolution using optical transition radiation, in Proceedings of the 2013 International Beam Instrumentation Conference, Oxford, UK, 2013 (JACoW, New York, 2013), WEAL2, p. 615 .

[22] B. Bolzon, A. Aryshev, T. Aumeyr, S. Boogert, P. Karataev, K. O. Kruchinin, T. Lefevre, S. Mazzoni, L. Nevay, M. Shevelev, N. Terunuma, J. Urakawa, and C. P. Welsch, Very high resolution optical transition radiation imaging system: Comparison between simulation and experiment, Phys. Rev. ST Accel. Beams 18, 082803 (2015).

[23] J. W. Goodman, Introduction to Fourier Optics, 3rd ed. (Roberts \& Company Publishers, Colorado, 2004).

[24] M. L. Ter-Mikaelian, High-Energy Electromagnetic Processes in Condensed Media (Wiley Interscience, New York, 1972).

[25] J.-C. Denard, P. Piot, K. Capek, and E. Feldl, High power beam profile monitor with optical transition radiation, in Proceedings of the Particle Accelerator Conference, PAC97, Vancouver, Canada, 1997 (IEEE, Piscataway, New Jersey, 1998), p. 2198.

[26] D. V. Karlovets and A. P. Potylitsyn, Transition radiation in the pre-wave zone for an oblique incidence of a particle on the flat target, Nucl. Instrum. Methods Phys. Res., Sect. B 266, 3738 (2008).

[27] M. Ross, S. Anderson, J. Frisch, K. Jobe, D. McCormick, B. McKee, J. Nelson, T. Smith, H. Hayano, T. Naito, and N. Terunuma, Very high resolution optical transition radiation beam profile monitor, AIP Conf. Proc. 648, 237 (2002).

[28] A. P. Potylitsyn, Transition radiation and diffraction radiation. Similarities and differences, Nucl. Instrum. Methods Phys. Res., Sect. B 145, 169 (1998).

[29] N. A. Potylitsina-Kube and X. Artru, Diffraction radiation from ultrarelativistic particles passing through a slit. Determination of the electron beam divergence, Nucl. Instrum. Methods Phys. Res., Sect. B 201, 172 (2003). 